\title{
Development of indirect competitive immuno-assay method using SPR detection for rapid and highly sensitive measurement of salivary cortisol levels
}

\author{
Yusuke Tahara ${ }^{1}$, Zhe Huang ${ }^{1}$, Tetsuro Kiritoshi ${ }^{1}$, Takeshi Onodera ${ }^{2}$ and Kiyoshi Toko ${ }^{1,2}$ * \\ Graduate School of Information Science and Electrical Engineering, Kyushu University, Fukuoka, Japan \\ ${ }^{2}$ Research and Development Center for Taste and Odor Sensing, Kyushu University, Fukuoka, Japan
}

Edited by:

Danilo Emilio De Rossi, University of Pisa, Italy

Reviewed by:

Dermot Diamond, Dublin City

University, Ireland

Francesca Montemurro, Research

Center E. Piaggio, Italy

*Correspondence:

Kiyoshi Toko, Graduate School of Information Science and Electrical Engineering, Kyushu University, 744 Motooka, Nishi-ku, Fukuoka

819-0395, Japan

e-mail: toko@ed.kyushu-u.ac.jp

\begin{abstract}
The monitoring of salivary cortisol as a key biomarker of an individual's stress response has been increasingly focused on. This paper describes the development of a novel cortisol immuno-assay method based on an indirect competitive method using a commercially available surface plasmon resonance instrument. The surface of an Au chip was modified with PEG6- $\mathrm{COOH}$ aromatic dialkanethiol self-assembled monolayers and hydrocortisone 3-(O-carboxymethyl) oxime (hydrocortisone 3-CMO) as a cortisol analog. A detection limit of $38 \mathrm{ppt}$ range with a measurement range of $10 \mathrm{ppt}-100 \mathrm{ppb}$ was accomplished without the incubation of a mixing solution consisting of standard cortisol and an anti-cortisol antibody, and the time for quantification of cortisol concentration was $8 \mathrm{~min}$ from the sample injection. We experimentally compared our immuno-assay with a commercialized salivary cortisol enzyme-linked immunosorbent assay (ELISA) kit using human saliva samples. It was found that the results obtained by the cortisol immuno-assay had a good correlation with those obtained by ELISA assay $(R=0.96)$. Our findings indicate the potential utility of the cortisol immuno-assay for measurements of human salivary cortisol levels.
\end{abstract}

Keywords: salivary cortisol, stress, surface plasmon resonance, self-assembled monolayer, immunosensor

\section{INTRODUCTION}

In the hypothalamus-pituitary-adrenal (HPA) axis, plasma, urinary, and salivary cortisol, key biomarkers of an individual's psychological stress response, are being increasingly focused on in psychobiological stress research (Kirschbaum and Hellhammer, 2007; Olff et al., 2007). Many research groups have reported the relationship between the cortisol concentration and stress-related disorders, including irritable bowel syndrome (IBS) (Patacchioli et al., 2001), chronic fatigue syndrome (CFS) (Jerjes et al., 2006), and posttraumatic stress disorder (PTSD) (Inslicht et al., 2006; Johnson et al., 2008).

Salivary cortisol, in particular, is a major neuroendocrine correlator of the neuroendocrine stress response and has been increasingly studied as a stress biomarker (Hellhammer et al., 2009). Salivary cortisol levels ranged between 0.1 and $10 \mathrm{ppb}(\mathrm{ng} / \mathrm{ml}$ ) (Aardal and Holm, 1995). Our research group has focused on salivary cortisol and its analytical method (Tahara et al., 2010), because saliva can be collected sufficient volumes for biochemical analyses, with some exceptions such as dry mouth patients, regardless of the time and place by a non-invasive, and stress-free process without medical personnel. The whole salivary secretion rate has been reported approximately $1 \mathrm{ml} / \mathrm{min}$ (resting saliva) (Molander and Birkhed, 1981). The salivary cortisol level differs greatly among individuals and with circadian variation (Adam et al., 2006). Other research groups have reported that salivary cortisol levels are significantly lower in IBS patients (Patacchioli et al., 2001) and that there is a significant difference between depressed and healthy people with regard to salivary cortisol levels when waking and $30 \mathrm{~min}$ after waking (Alderling et al., 2008). However, immuno-assay-based clinical auto analytical instruments are expensive and their use is time-consuming. Conventional enzyme-linked immunosorbent assay (ELISA) in labs usually takes approximately $4 \mathrm{~h}$ to complete the analysis and multiple steps are required. Thus, the development of rapid, portable, and easy-to-use analytical devices designed to measure and rapidly report cortisol levels would have a great impact on future research and the treatment of people with stressrelated disorders. Recently, various types of cortisol biosensors for point-of-care testing (POCT), such as a polyaniline based electrochemical immunosensor (Arya et al., 2011; Kaushik et al., 2013; Vasudev et al., 2013), an immunosensor with a fluid control mechanism (Yamaguchi et al., 2013), a single-walled carbon nanotubes (SWNT) chemiresistor transducer-based cortisol immunosensor (Tlili et al., 2011) have been proposed.

Our research group has developed a compact sensing system using surface plasmon resonance (SPR) immunosensors for the on-site detection of trinitrotoluene (MW: 227.1) (Mizuta et al., 2008; Onodera et al., 2009; Onodera, 2013). SPR immunosensors are realized by combining SPR sensors, which can be used to detect changes in the refractive index of the sensor surface with high-sensitivity, with an antigen-antibody interaction, resulting in high selectivity. However, cortisol, a steroid hormone, is a monovalent antigen with a low molecular weight (362.47). Therefore, the sandwich immuno-assay method cannot be applied. Other research groups have reported an SPR immunosensor using a direct competitive assay with primary and secondary antibodies used for the amplification of signals [response time: $15 \mathrm{~min}$ 
from sample injection, assay dynamic range: 91-900 ppt, detection limit (the blank less two SDs of the blank) using standard solution: 13 ppt] (Mitchell et al., 2009), using a direct assay with polycarboxylate-hydrogel-based coating for the antibody immobilization (response time: flowed over the sensors surface for $15 \mathrm{~min}$, linear detection range: 5-154 ppb, detection limit using standard solution: $10 \mathrm{ppb}$ ) (Frasconi et al., 2009), as well as an SPR immunosensor using a cortisol-conjugated BSA-modified Au chip as a cortisol analog (response time: $15 \mathrm{~min}$ from sample injection, detection range: $1.5-10 \mathrm{ppb}$, detection limit using standard solution: $360 \mathrm{ppt}$ ) (Stevens et al., 2008).

In this study, we report a salivary cortisol immuno-assay method using a commercially available SPR instrument and a newly designed indirect competitive method for the quantitative evaluation of human psychosomatic stress. To this end, we modified a sensor surface with PEG6-COOH aromatic dialkanethiol self-assembled monolayers (SAMs) and hydrocortisone 3-CMO as a cortisol analog for highly sensitive, accurate, and rapid detection. Moreover, we compared the characteristics of the cortisol immuno-assay method a conventional ELISA assay using human saliva.

\section{MATERIALS AND METHODS REAGENTS AND CHEMICALS}

Mouse anti-cortisol monoclonal antibody (association constant $K_{\mathrm{A}}=1.7 \times 10^{9} \mathrm{M}^{-1}$, cortisol antibody) was purchased from Abcam PLC (UK). As the cortisol analog, hydrocortisone 3-( $O$ carboxymethyl) oxime (hydrocortisone 3-CMO) was purchased from Sigma Aldrich (USA). PEG6-COOH aromatic dialkanethiol (PEG6-COOH) was purchased from SensoPath Technologies, Inc. (USA), and was used as a reagent to form SAMs on the chip surface. The Au sensor chip included in the SIA Kit, the $N$-ethyl$N$-(3-dimethylaminopropyl) carbodiimide hydrochloride (EDC) included in an amine coupling kit, the $N$-hydroxysuccinimide (NHS) for sensor surface fabrication, a borate buffer $(\mathrm{pH} 8.5$, $10 \mathrm{mM}$ disodium tetraborate, $1 \mathrm{M} \mathrm{NaCl}$ ), and $50 \mathrm{mM} \mathrm{NaOH}$ were purchased from GE Healthcare Bio-Science AB (Sweden). Ethylenediamine was purchased from Wako Pure Chemical Industries, Ltd. (Japan). An ELISA kit was used as a conventional salivary cortisol detection method, and Salimetrics oral swabs, used as a salivary collection material were purchased from Salimetrics LLC (USA). Water purified using a Milli-Q integral water purification system was used as the solvent. All aqueous solutions were prepared with Milli-Q water obtained from a Milli-Q system (Millipore Corporation, USA).

\section{MODIFICATION OF SENSOR CHIP SURFACE}

As a preliminary cleaning step, an Au sensor chip covered with a 50-nm-thick layer of unmodified gold was first ultrasonically cleaned in acetone for $10 \mathrm{~min}$, ethanol for $2 \mathrm{~min}$, and 2-propanol for $2 \mathrm{~min}$. Subsequently, the sensor surface was cleaned in standard clean solution (a mixture of ammonia solution, hydrogen peroxide solution, and pure water with a ratio of $1: 1: 5$ ) heated to $90^{\circ} \mathrm{C}$ for $20 \mathrm{~min}$. Modification of the sensor chip surface was performed as follows (Figure 1): the sensing surface of the Au sensor chip was immersed in $1 \mathrm{mM}$ PEG6-COOH (Lahiri et al., 1999) in ethanol for $24 \mathrm{~h}$ to form SAMs on the sensor surface. After cleaning with ethanol by ultrasonication, a mixture solution consisting of $0.2 \mathrm{M}$ EDC in water and $50 \mathrm{mM}$ NHS in water was added dropwise onto the sensor surface and incubated for $30 \mathrm{~min}$ to activate the terminal carboxyl group. Next, ethylenediamine in the $\mathrm{pH} 8.5$ borate buffer was added dropwise onto the sensor surface and incubated for $30 \mathrm{~min}$ to induce amine coupling by covering the terminal carboxyl group of the SAM into an amino group. Simultaneously, $10 \mathrm{mM}$ hydrocortisone 3-CMO in DMF with a carboxyl group as a cortisol analog, 0.4 M EDC in water, and 0.1 M NHS in DMF were mixed with a volume ratio of $1: 1: 1$ and incubated for $60 \mathrm{~min}$ to activate the carboxyl group of the cortisol analog. The mixed solution was added dropwise onto the sensor surface and incubated for $60 \mathrm{~min}$. Finally, the cortisol analog was bound to ethylenediamine immobilized on the film surface as a result of amine coupling.

\section{CORTISOL DETECTION USING SPR IMMUNOSENSOR BY INDIRECT COMPETITIVE ASSAY}

Cortisol detection was performed by SPR using a Biacore J system (GE Healthcare, Japan). An indirect competitive assay was applied for cortisol measurements. $10 \mathrm{mM}$ 4-(2-hydroxyethyl)1-piperazineethanesulfonic acid (HEPES) buffered saline (HBS, $150 \mathrm{mM} \mathrm{NaCl}, 0.05 \%$ Tween 20 , pH 7.4) was used as the running buffer.

When the antibody solution is injected onto the chip surface, the antibodies bind to the cortisol analog immobilized onto the chip surface, resulting in an increased sensor response. The time from injection to reach the sensor surface is within a few seconds. The increase in the sensor response $\left(\Delta \theta_{0}\right)$ represents the number of antibodies bound to the chip surface. When a mixture of antibodies and cortisol is injected onto the chip surface, the antibodies that have already bound to the cortisol do not bind to the cortisol analog on the chip surface. Namely, the cortisol in the solution inhibits the antibodies from binding to the chip surface. Therefore, the concentration of antibodies bound to the chip surface decreases from that when only the antibodies are injected $\left(\Delta \theta_{1}\right)$. It is considered that $\Delta \theta_{1}$ decreases as the percentage of cortisol in the mixture increases. Here, the relative change in the concentration of antibodies bound to the chip surface, i.e., the bound percentage, is calculated by

$$
\left(\Delta \theta_{1} / \Delta \theta_{0}\right) \times 100 \text {. }
$$

The smaller the bound percentage, the larger the concentration of antibodies bound to cortisol. Antibodies and cortisol, each with a concentration of twice the final concentration, were prepared for the measurement.

A mixture of the antibody solution and HBS buffer was injected onto the chip surface for $5 \mathrm{~min}$, followed by the injection of a regeneration solution for $3 \mathrm{~min}$ at a flow rate of $15 \mu \mathrm{l} / \mathrm{min}$ to regenerate the chip surface. Subsequently, the antibody and cortisol solutions were mixed with a ratio of $1: 1$ and injected onto the chip surface for $5 \mathrm{~min}$ at a flow rate of $15 \mu \mathrm{l} / \mathrm{min}$. Next, to regenerate the sensor surface, $50 \mathrm{mM} \mathrm{NaOH}$ was allowed to flow over the sensor surface for $3 \mathrm{~min}$ at a flow rate of $15 \mu \mathrm{l} / \mathrm{min}$.

\section{CALCULATION OF ASSOCIATION CONSTANT}

Antibody solutions with different concentrations (0.1-2.0 ppm) were injected onto the three sensor chips with the cortisol analog 

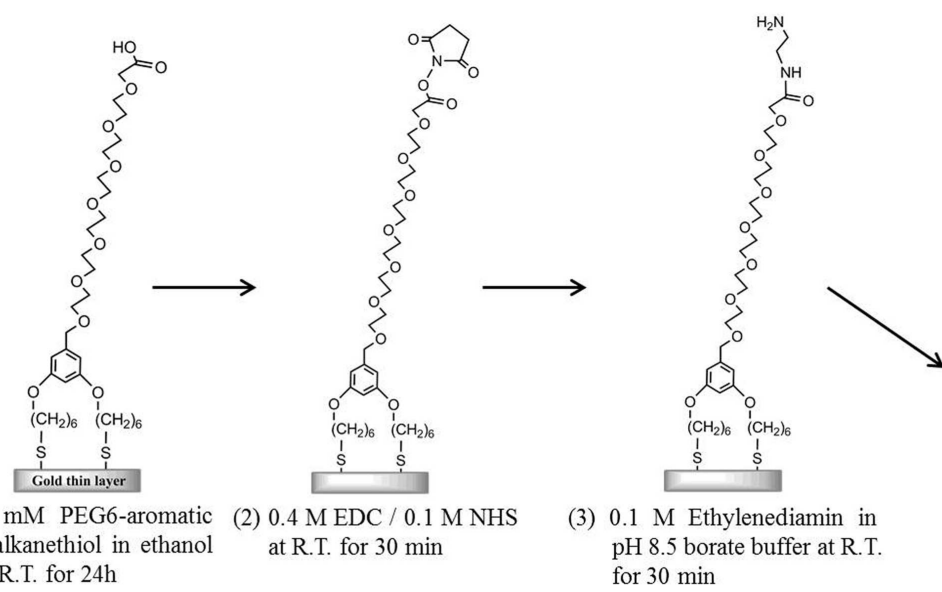

(1) $1 \mathrm{mM}$ PEG6-aromatic dialkanethiol in ethanol at R.T. for $24 \mathrm{~h}$

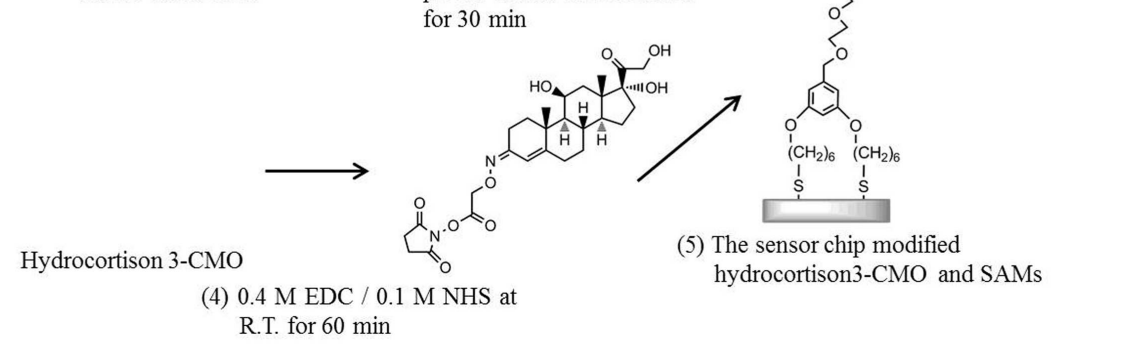

$0.1 \mathrm{M}$ Ethylenediamin in $\mathrm{pH} 8.5$ borate buffer at R.T. for $30 \mathrm{~min}$

R.T. for $60 \mathrm{~min}$

FIGURE 1 | Fabrication procedure of the sensor chip modified with cortisol analog

immobilized on the surface for $5 \mathrm{~min}$ at $30 \mu \mathrm{L} / \mathrm{min}$. After the injection, the sensor chip was incubated for 5 min to allow spontaneous dissociation. The obtained sensorgram was used to calculate the reaction rate constants. BIAevaluation version 4.1 software (GE Healthcare Bio-Science) was used for analysis as well as the "bivalent with mass transfer" fitting model, which assumes that antibodies undergo bivalent binding and that the association of antibodies occurs under mass transport limitation. Five sensorgrams with six concentrations of the antibody solution were simultaneously analyzed by the fitting program to calculate the association rate constant $K_{\mathrm{a}}$ and the dissociation rate constant $K_{\mathrm{d}}$. Using these constants, the association constant $K_{\mathrm{A}}\left(=K_{\mathrm{a}} / K_{\mathrm{d}}\right)$ was calculated.

\section{CALIBRATION CURVE FOR CORTISOL DETECTION}

To construct a calibration curve, the sensor response was measured three times for each cortisol solution with concentrations of $0.01-$ $100 \mathrm{ppb}(1 \mathrm{ppb}=1 \mu \mathrm{g} / \mathrm{l}=2.8 \mathrm{nM})$ following the above procedure. A bound percentage equal to a value three times the standard deviation was adopted as the detection limit. BIAevaluation version 4.1 software (GE Healthcare Bio-Science) was used for calculating the calibration curves.

\section{COLLECTION AND PREPARATION OF HUMAN SALIVA SAMPLES}

To collect human saliva, four healthy young Japanese adults ( $25 \pm 1.2$ years) were enrolled in this study. The study protocol was approved by the Ethical Committee of Kyushu University. The subjects were not allowed to take any food or drinks, except for water, for $1 \mathrm{~h}$ prior to the collection. To clean the oral cavity, each subject rinsed their mouth. To collect whole saliva, the subjects inserted a salimetrics oral swab into the oral cavity under the tongue for about $3 \mathrm{~min}$. Saliva was collected at 10:00 a.m., 2:00, and 6:00 p.m. The saliva was separated from the oral swab by centrifugalization at 3,000 rpm for $15 \mathrm{~min}$. Then, the saliva samples were stored at $-20^{\circ} \mathrm{C}$ until analysis.

\section{RESULTS AND DISCUSSION} EVALUATION OF ASSOCIATION CONSTANTS OF THE CORTISOL ANALOG TO THE ANTIBODY

In order to confirm that the hydrocortisone 3-( $O$-carboxymethyl) oxime (hydrocortisone 3-CMO) can be applied to this indirect competitive assay as a cortisol analog, the strength of association constants $\left(K_{\mathrm{A}}\right)$ of the modified sensor chip was analyzed. From the calculation result, $K_{\mathrm{A}}$ was $3.07 \times 10^{7} \mathrm{M}^{-1}$ and lower than $K_{\mathrm{A}}$ between cortisol and the cortisol antibody $\left(K_{\mathrm{A}}=1.7 \times 10^{9} \mathrm{M}^{-1}\right)$. It indicated that the cortisol analog-modified sensor chip can be applied to the indirect competitive assay.

\section{CALIBRATION CURVE}

A calibration curve for the cortisol measurement was obtained using a standard cortisol solution consisting of cortisol with various concentrations, HBS, and $2.5 \%$ ethanol $(n=3)$. Figure 2 shows an SPR sensorgram obtained by this measurement. When a mixture of $600 \mathrm{ppb}$ antibody and HBS (final concentration, $300 \mathrm{ppb}$ ) was injected onto the chip, the change in the sensor response $\left(\Delta \theta_{1}\right)$ was $207.4 \pm 4.7 \mathrm{RU}(n=18)$. Here, 1000 RU corresponds to approximately $1 \mathrm{ng} / \mathrm{mm}^{2}$, and the amount of immobilized antibody was $207.4 \mathrm{pg} / \mathrm{mm}^{2}$. Dissociation of the bound antibody from the sensing surface is required for the next immunocycle. These results indicate that the regeneration 


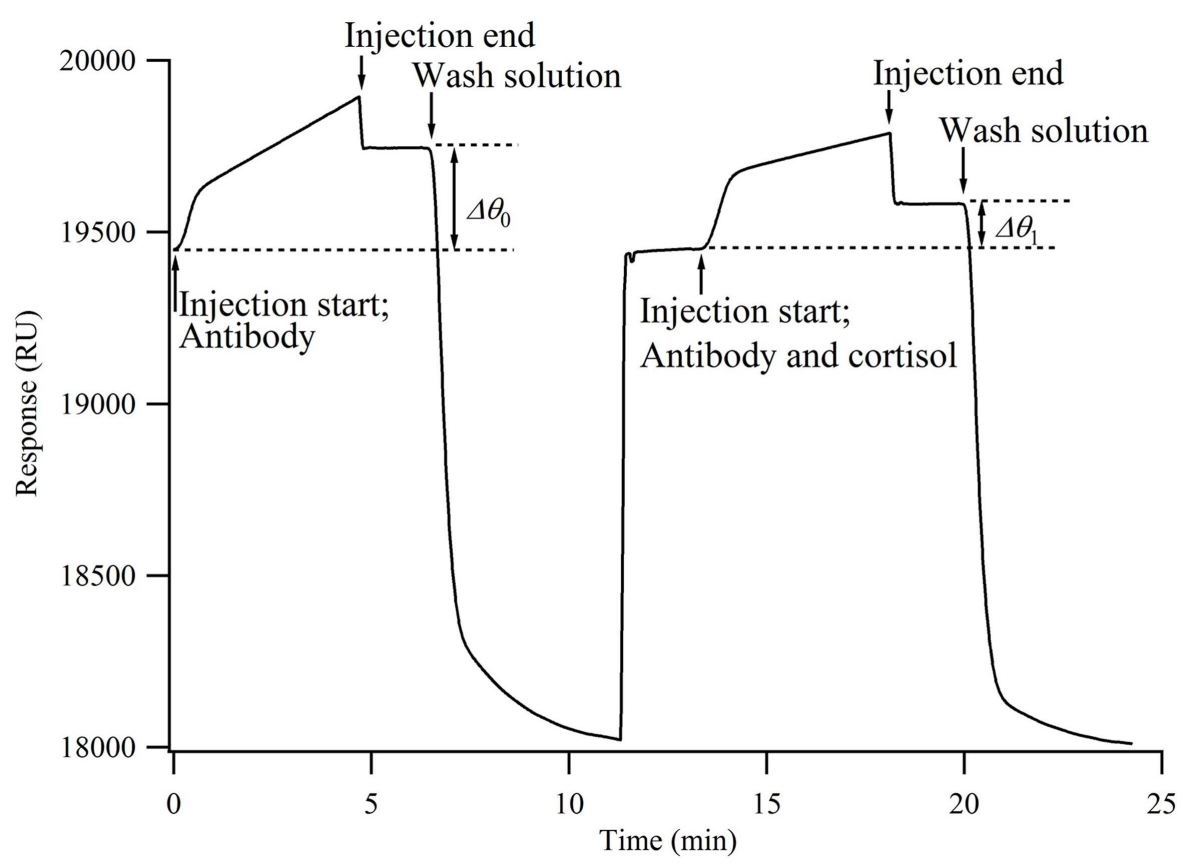

FIGURE 2 | Surface plasmon resonance sensorgram obtained from indirect competitive assay

conditions are suitable, because the sensor responses $\left(\Delta \theta_{1}\right)$ were nearly unchanged.

Before injection onto the sensor surface, each mixture of the antibody and cortisol solutions was incubated for $2 \mathrm{~h}$. A mixture solution containing $600 \mathrm{ppb}$ antibody and the each standard cortisol solution was applied for $5 \mathrm{~min}$ after incubation for $2 \mathrm{~h}$ or without incubation before injection. Figure 3 shows the obtained calibration curves. Even without incubation, a calibration curve similar to that for the case of $2 \mathrm{~h}$ incubation was obtained. The detection limits of the calibration curves in the case of $2 \mathrm{~h}$ incubation and no incubation were 72.8 and $38.0 \mathrm{ppt}$, respectively. Alderling et al. reported that the cortisol level in human saliva ranges from approximately 2-20 nM (0.7-7.1 ppb) with circadian variation. Thus, this cortisol immuno-assay method does not need sample incubation, antibody, and cortisol solution, before sample injection to monitor human cortisol levels. Here, $\Delta \theta_{1}$ for cortisol quantification was determined approximately $8 \mathrm{~min}$ after sample injection and rapid compared with other reports. Moreover, it can be used to analyze the cortisol concentrations in blood plasma and urine, which are approximately 10 times higher than that in saliva, upon controlling the sensitivity.

\section{COMPARISON OF SPR AND ELISA ASSAY}

To evaluate the accuracy of the cortisol biosensor for detecting cortisol in human saliva, we carried out a comparison with a conventional cortisol ELISA kit. The calibration curve for the cortisol measurement was applied using standard cortisol solution included in a commercialized ELISA kit $(n=2)$. Figure 4 shows the calculated cortisol values using the sensor and ELISA applied to cortisol standard solution $(0.12-3 \mathrm{ppb})$. The values of

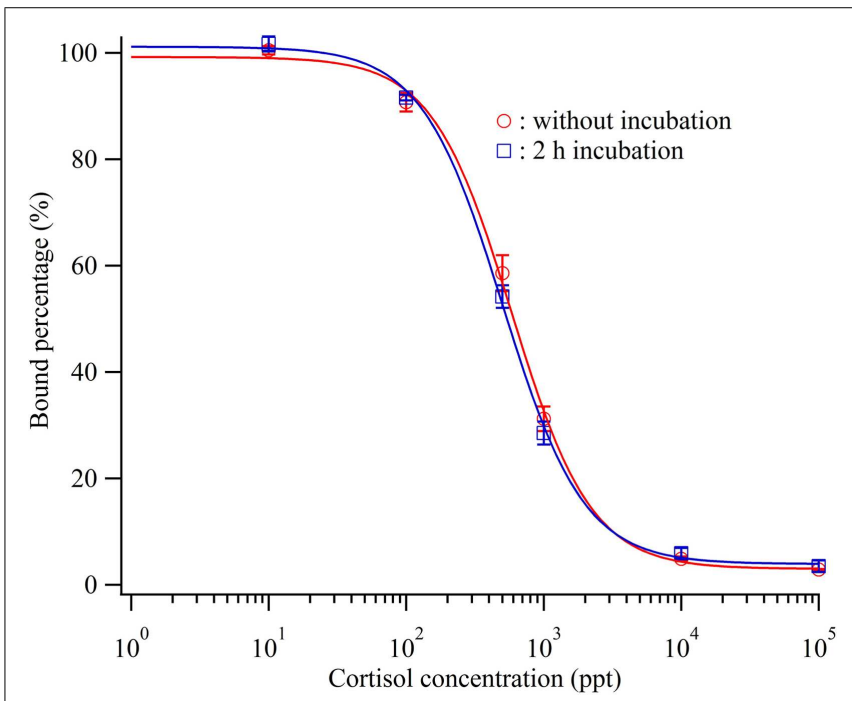

FIGURE 3 | Calibration curve of the cortisol biosensor with or without incubation before injection of cortisol standard cortisol and cortisol antibody. C; without incubation, $\square ; 2$ h incubation.

determination $\left(R^{2}\right)$ of the sensor and ELISA were 0.98 and 0.99 , respectively.

In the ELISA assay, after the human saliva samples were thawed at room temperature, they were centrifuged at 3,000 rpm for $15 \mathrm{~min}$ and the supernatant sample was used in the ELISA assay. The measurement was performed following the manufacturer's manual. The cortisol concentration ranged between 1.3 and 


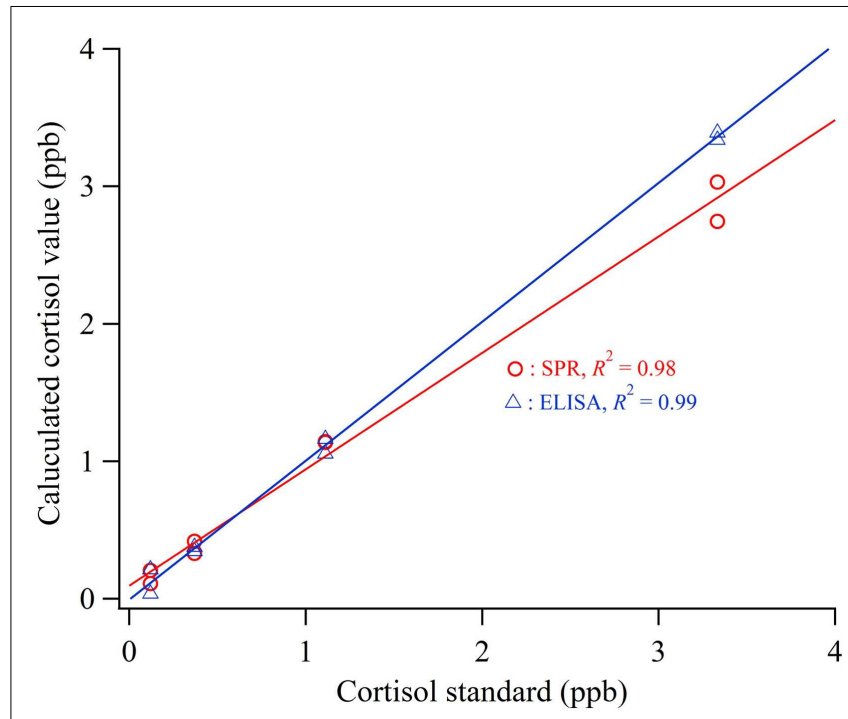

FIGURE 4 | Calculated cortisol values using the sensor and ELISA applied to cortisol standard solution.
4.1 ppb (3.7 and 11.2 $\mathrm{nM})$. These results were shown to be in reasonable agreement with those previously reported (Kirschbaum and Hellhammer, 2007; Hellhammer et al., 2009). In the SPR assay, after the human saliva samples were thawed at room temperature, they were centrifuged at $13,000 \mathrm{rpm}$ using a Microcon centrifugal filter unit membrane (molecular weight cutoff $30 \mathrm{kDa}$, Millipore Corporation, USA) and mixed with HBS with a volume ratio of 1:3 to remove proteins. The SPR assay was carried out following the above procedure. Here, the cross reactivity of the anti-cortisol antibody to steroid hormones in saliva such as progesterone, testosterone, estradiol, and estriol was lower than $0.1 \%$ according to the data sheet of the manufacturers.

Figure 5 shows the results of the comparative experiment using human saliva samples. Linear regression analysis was used to determine the relationship between the measurement values obtained by the biosensor and the ELISA. The correlation coefficient was 0.96 and the regression equation relative the values of cortisol obtained using the SPR (Cortisol SPR $_{\text {) }}$ and ELISA (Cortisol ELISA) $_{\text {) }}$ was Cortisol $_{\text {ELISA }}=0.96 \times$ Cortisol $_{\mathrm{SPR}}+0.339$. The values of slope $\pm 95 \%$ confidence interval (CI) and intercept $\pm 95 \%$ were $0.96 \pm 0.239$ and $0.339 \pm 0.572$, respectively. Thus, it is indicated that the cortisol immuno-assay has good correlation and is capable of performing with similar accuracy to the conventional ELISA. It is necessary to develop the technology for improving sample preparation method in a short time.

\section{CONCLUSION}

A cortisol immuno-assay employing a newly designed indirect competitive method and a commercially available SPR instrument with high-sensitivity, rapid, simple, and label-free measurements was invented for salivary cortisol measurements to replace timeconsuming laboratory analysis and facilitate the POCT of salivary cortisol. The Au surface of a sensor chip was modified with PEG6$\mathrm{COOH}$ aromatic dialkanethiol using hydrocortisone 3-CMO as a

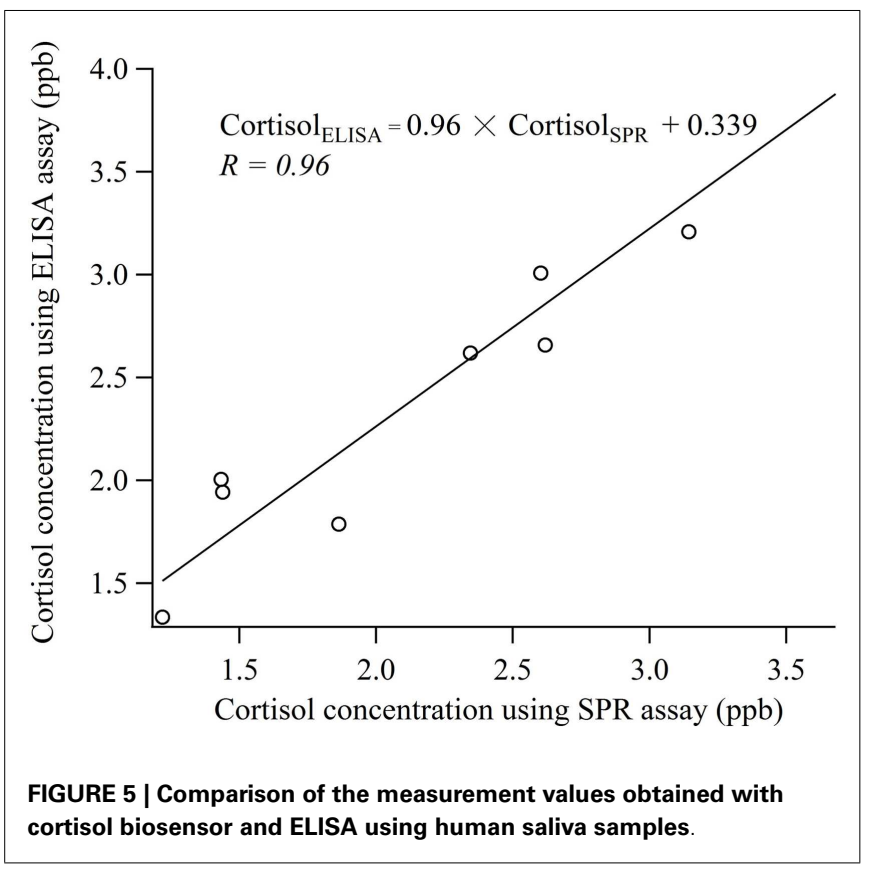

cortisol analog. A detection limit of 38 ppt was achieved by the sensor and the response time was $5 \mathrm{~min}$ from the sample injection. Moreover, because there was no effect of the incubation time of standard cortisol and cortisol antibody, cortisol measurement was possible without incubation. We experimentally compared our immuno-assay method using the commercially available SPR instrument with a commercialized salivary cortisol ELISA kit using human saliva samples. The results obtained by the cortisol biosensor had a good correlation with those obtained by ELISA assay $(R=0.96)$. Therefore, the measurement results suggest that the biosensor can be used to monitor salivary cortisol levels of human saliva samples. Moreover, it can be used to analyze the cortisol concentrations in blood plasma and urine.

\section{REFERENCES}

Aardal, E., and Holm, A. C. (1995). Cortisol in saliva - reference ranges and relation to cortisol in serum. Eur. J. Clin. Chem. Clin. Biochem. 33, 927-932.

Adam, E. K., Hawkley, L. C., Kudielka, B. M., and Cacioppo, J. T. (2006). Day-to-day dynamics of experience-cortisol associations in a population-based sample of older adults. Proc. Natl. Acad. Sci. U.S.A. 103, 17058-17063. doi:10.1073/pnas. 0605053103

Alderling, M., De La Torre, B., Forsell, Y., Lundberg, I., Söndergaard, H. P., and Theorell, T. (2008). Psychiatric diagnoses and circadian saliva cortisol variations in a Swedish population-based sample (the PART study). Psychother. Psychosom. 77, 129-131. doi:10.1159/000112892

Arya, S. K., Dey, A., and Bhansali, S. (2011). Polyaniline protected gold nanoparticles based mediator and label free electrochemical cortisol biosensor. Biosens. Bioelectron. 28, 166-173. doi:10.1016/j.bios.2011.07.015

Frasconi, M., Mazzarino, M., Botrè, F., and Mazzei, F. (2009). Surface plasmon resonance immunosensor for cortisol and cortisone determination. Anal. Bioanal. Chem. 394, 2151-2159. doi:10.1007/s00216-009-2914-6

Hellhammer, D., Wust, S., and Kudielka, B. (2009). Salivary cortisol as a biomarker in stress research. Psychoneuroendocrinology 34, 163-171. doi:10.1016/j.psyneuen. 2008.10.026

Inslicht, S. S., Marmar, C. R., Neylan, T. C., Metzler, T. J., Hart, S. L., Otte, C., et al. (2006). Increased cortisol in women with intimate partner violencerelated posttraumatic stress disorder. Psychoneuroendocrinology 31, 825-838. doi:10.1016/j.psyneuen.2006.08.002 
Jerjes, W. K., Peters, T. J., Taylor, N. F., Wood, P. J., Wessely, S., and Cleare, A. J. (2006). Diurnal excretion of urinary cortisol, cortisone, and cortisol metabolites in chronic fatigue syndrome. J. Psychosom. Res. 60, 145-153. doi:10.1016/j. jpsychores.2006.03.008

Johnson, D. M., Delahanty, D. L., and Pinna, K. (2008). The cortisol awakening response as a function of PTSD severity and abuse chronicity in sheltered battered women. J. Anxiety Disord. 22, 793-800. doi:10.1016/j.janxdis.2007.08.006

Kaushik, A., Vasudev, A., Arya, S. K., and Bhansali, S. (2013). Mediator and label free estimation of stress biomarker using electrophoretically deposited Ag@AgO-polyaniline hybrid nanocomposite. Biosens. Bioelectron. 50, 35-41. doi:10.1016/j.bios.2013.06.012

Kirschbaum, C., and Hellhammer, D. H. (2007). "Salivary cortisol," in Encyclopedia of Stress, 2nd Edn, ed. G. Fink (New York: Academic Press), 405-409.

Lahiri, J., Isaacs, L., Tien, J., and Whitesides, G. M. (1999). A strategy for the generation of surfaces presenting ligands for studies of binding based on an active ester as a common reactive intermediate: a surface plasmon resonance study. Anal. Chem. 71, 777-790. doi:10.1021/ac980959t

Mitchell, J. S., Lowe, T. E., and Ingram, J. R. (2009). Rapid ultrasensitive measurement of salivary cortisol using nano-linker chemistry coupled with surface plasmon resonance detection. Analyst 134, 380-386. doi:10.1039/b817083p

Mizuta, Y., Onodera, T., Singh, P., Matsumoto, K., Miura, N., and Toko, K. (2008). Development of an oligo(ethylene glycol)-based SPR immunosensor for TNT detection. Biosens. Bioelectron. 24, 191-197. doi:10.1016/j.bios.2008.03.042

Molander, L., and Birkhed, D. (1981). Effect of single oral doses of various neuroleptic drugs on salivary secretion rate, $\mathrm{pH}$, and buffer capacity in healthy subjects. Psychopharmacology 75, 114-118. doi:10.1007/bf00432171

Olff, M., De Vries, G.-J., Güzelcan, Y., Assies, J., and Gersons, B. P. R. (2007). Changes in cortisol and DHEA plasma levels after psychotherapy for PTSD. Psychoneuroendocrinology 32, 619-626. doi:10.1016/j.psyneuen.2007.04.001

Onodera, T. (2013). "Electronic dog nose using surface plasmon resonance method," in Biochemical Sensors, ed. K. Toko (Singapore: Pan Stanford Publishing), 351-375.

Onodera, T., Miura, N., Matsumoto, K., and Toko, K. (2009). "Development of an 'electronic dog nose' based on an SPR immunosensor for highly sensitive detection of explosives," in Anti-Personnel Landmine Detection for Humanitarian Demining, eds K. Furuta and J. Ishikawa (London: Springer), 193-205.
Patacchioli, F. R., Angelucci, L., Dellerba, G., Monnazzi, P., and Leri, O. (2001). Actual stress, psychopathology and salivary cortisol levels in the irritable bowel syndrome (IBS). J. Endocrinol. Invest. 24, 173-177. doi:10.1007/BF03343838

Stevens, R. C., Soelberg, S. D., Near, S., and Furlong, C. E. (2008). Detection of cortisol in saliva with a flow-filtered, portable surface plasmon resonance biosensor system. Anal. Chem. 80, 6747-6751. doi:10.1021/ac800892h

Tahara, Y., Yoshikawa, S., Shetty, V., and Yamaguchi, M. (2010). Immunochromatographic cortisol biosensor using enzyme-labeled conjugate. Sens. Mater. 22, 427-437.

Tlili, C., Myung, N. V., Shetty, V., and Mulchandani, A. (2011). Label-free, chemiresistor immunosensor for stress biomarker cortisol in saliva. Biosens. Bioelectron. 26, 4382-4386. doi:10.1016/j.bios.2011.04.045

Vasudev, A., Kaushik, A., Tomizawa, Y., Norena, N., and Bhansali, S. (2013). An LTCC-based microfluidic system for label-free, electrochemical detection of cortisol. Sens. Actuators B Chem. 182, 139-146. doi:10.1016/j.snb.2013.02.096

Yamaguchi, M., Matsuda, Y., Sasaki, S., Sasaki, M., Kadoma, Y., Imai, Y., et al. (2013). Immunosensor with fluid control mechanism for salivary cortisol analysis. Biosens. Bioelectron. 41, 186-191. doi:10.1016/j.bios.2012.08.016

Conflict of Interest Statement: The authors declare that the research was conducted in the absence of any commercial or financial relationships that could be construed as a potential conflict of interest.

Received: 06 December 2013; accepted: 07 May 2014; published online: 30 May 2014. Citation: Tahara Y, Huang Z, Kiritoshi T, Onodera T and Toko K (2014) Development of indirect competitive immuno-assay method using SPR detection for rapid and highly sensitive measurement of salivary cortisol levels. Front. Bioeng. Biotechnol. 2:15. doi: 10.3389/fbioe.2014.00015

This article was submitted to Bionics and Biomimetics, a section of the journal Frontiers in Bioengineering and Biotechnology.

Copyright (c) 2014 Tahara, Huang, Kiritoshi, Onodera and Toko. This is an open-access article distributed under the terms of the Creative Commons Attribution License (CC $B Y)$. The use, distribution or reproduction in other forums is permitted, provided the original author(s) or licensor are credited and that the original publication in this journal is cited, in accordance with accepted academic practice. No use, distribution or reproduction is permitted which does not comply with these terms. 